\title{
UNCONDITIONAL CONVERGENCE IN THE STRONG OPERATOR TOPOLOGY AND $\ell_{\infty}$
}

\author{
IOANA GHENCIU \\ University of Wisconsin - River Falls, Department of Mathematics, River Falls, WI 54022-5001 \\ e-mail: ioana.ghenciu@uwrf.edu \\ and PAUL LEWIS \\ University of North Texas, Department of Mathematics, Box 311430, Denton, Texas 76203-1430 \\ e-mail:lewis@unt.edu
}

(Received 26 May 2010; accepted 30 November 2010; first published online 10 March 2011)

\begin{abstract}
In this paper we study non-complemented spaces of operators and the embeddability of $\ell_{\infty}$ in the spaces of operators $L(X, Y), K(X, Y)$ and $K_{w^{*}}\left(X^{*}, Y\right)$. Results of Bator and Lewis [2, 3] (Bull. Pol. Acad. Sci. Math. 50(4) (2002), 413-416; Bull. Pol. Acad. Sci. Math. 549(1) (2006), 63-73), Emmanuele [8-10] (J. Funct. Anal. 99 (1991), 125-130; Math. Proc. Camb. Phil. Soc. 111 (1992), 331-335; Atti. Sem. Mat. Fis. Univ. Modena 42(1) (1994), 123-133), Feder [11] (Canad. Math. Bull. 25 (1982), 78-81) and Kalton [16] (Math. Ann. 208 (1974), 267-278), are generalised. A vector measure result is used to study the complementation of the spaces $W(X, Y)$ and $K(X, Y)$ in the space $L(X, Y)$, as well as the complementation of $K(X, Y)$ in $W(X, Y)$. A fundamental result of Drewnowski [7] (Math. Proc. Camb. Phil. Soc. 108 (1990), 523-526) is used to establish a result for operator-valued measures, from which we obtain as corollaries the Vitali-Hahn-Saks-Nikodym theorem, the Nikodym Boundedness theorem and a Banach space version of the Phillips Lemma.
\end{abstract}

2010 Mathematics Subject Classification. 46B05, 46B28, 46B25.

1. Introduction. Let $\left(e_{n}\right)$ denote the unit vector basis of $c_{0} . E, F, X, Y$ will denote real Banach spaces. The set of all continuous linear transformations from $E$ to $F$ will be denoted by $L(E, F)$, and the compact (respectively, weakly compact) operators will be denoted by $K(E, F)$ (resp. $W(E, F)$ ). The $w^{*}-w$ continuous maps from $E^{*}$ to $F$ (resp. $w^{*}-w$ continuous compact) will be denoted by $L_{w^{*}}\left(E^{*}, F\right)\left(\operatorname{resp} . K_{w^{*}}\left(E^{*}, F\right)\right)$. If $\left(T_{n}\right)$ is a sequence in $L(E, F)$, then $\left(T_{n}\right) \rightarrow T$ in the strong operator topology (sot) if $\left(T_{n}(x)\right)$ converges to $T(x)$ for all $x \in E$ and $\left(T_{n}\right) \rightarrow T$ in the weak operator topology (wot) if $\left(T_{n}(x)\right)$ converges weakly to $T(x)$ for all $x \in E$.

We note that $X \simeq L(\mathbb{R}, X)$. Let $\Sigma$ be a $\sigma$-algebra of subsets of $\Omega$. If $\left(A_{i}\right)_{i=1}^{\infty}$ is a sequence from $\Sigma$, then $\sigma\left(\left(A_{i}\right)\right)$ denotes the $\sigma$-algebra of subsets of $\cup_{i=1}^{\infty} A_{i}$ generated by $\left(A_{i}\right)_{i=1}^{\infty}$. If this sequence is pairwise disjoint, we can identify $\sigma\left(\left(A_{i}\right)\right)$ with $\mathcal{P}$, the power class of the positive integers. If $\mathcal{A}$ is an algebra of subsets of $\Omega$, a bounded and finitely additive set function $\mu: \mathcal{A} \rightarrow X$ is said to be strongly additive if $\left\|\mu\left(A_{i}\right)\right\| \rightarrow 0$, whenever $\left(A_{i}\right)$ is a pairwise disjoint sequence from $\mathcal{A}$. Equivalently, a bounded and finitely additive set function $\mu: \mathcal{A} \rightarrow X$ is strongly additive iff there is a finitely additive non-negative real valued measure $\lambda$ so that $\mu \ll \lambda[6$, Corollary 5.3, p. 28]. Drewnowski [7, p. 38] showed that if $\mu: \Sigma \rightarrow X$ is strongly additive and $\left(A_{i}\right)$ is a pairwise disjoint 
sequence from $\Sigma$, then there is a subsequence $\left(B_{i}\right)$ of $\left(A_{i}\right)$ so that $\mu: \sigma\left(\left(B_{i}\right)\right) \rightarrow X$ is countably additive. A sequence $\left(\mu_{i}\right)$ of strongly additive measures on $\mathcal{A}$ is said to be uniformly strongly additive if for any pairwise disjoint sequence $\left(A_{n}\right)$ in $\mathcal{A}$, $\lim _{n}\left\|\mu_{i}\left(A_{n}\right)\right\|=0$ uniformly in $i$.

The Diestel-Faires theorem [6, p. 20] is helpful in identifying isomorphic copies of both $c_{o}$ and $\ell_{\infty}$. The key ingredient in the proof of the Diestel-Faires theorem is a fundamental lemma by Rosenthal [22]. The proof of this lemma, which appears in [6], is from Kupka [17] and is described as an elegant simplification of Rosenthal's original argument. The proof of the algebra version is based on the proof of the $\sigma$-algebra version, the Hahn-Banach theorem and isomorphic identifications. We present an elementary proof of Rosenthal's lemma that handles both cases simultaneously. (In the $\sigma$-algebra case, replace infinite sums with unions.)

THEOREM 1 ( Rosenthal's Lemma [22]). Suppose that $\mathcal{A}$ is an algebra of subsets of $\Omega$, and $\left(\mu_{n}\right)$ is a uniformly bounded sequence of finitely additive real-valued measures defined on $\mathcal{A}$. If $\left(A_{n}\right)$ is a pairwise disjoint sequence of sets in $\mathcal{A}$ and $\epsilon>0$, then there is a subsequence $\left(A_{n_{j}}\right)$ of $\left(A_{n}\right)$ such that

$$
\sum_{j \neq i}\left|\mu_{n_{i}}\right|\left(A_{n_{j}}\right)<\epsilon
$$

for each $i \in \mathbb{N}$.

Proof. Let $\epsilon>0$, and let $\left(A_{i}\right)$ be a pairwise disjoint sequence of sets in $\mathcal{A}$. Since $\left(\left|\mu_{i}\right|\right)$ is bounded, using a diagonal process we may assume without loss of generality that $x_{i}=\lim _{n}\left|\mu_{n}\right|\left(A_{i}\right)$ exists for all $i$.

We claim that $\lim _{k} x_{k}=0$. Suppose not. Without loss of generality $x_{k}>\delta$ for all $k$, for some $\delta>0$. Let $M>0$ so that $\left|\mu_{n}\right|<M$ for all $n$ and let $N \in \mathbb{N}$ so that $\frac{N \delta}{2}>M$. Since $\lim _{n}\left|\mu_{n}\right|\left(A_{k}\right)>\delta$ for all $k$, one can find an $n \in \mathbb{N}$ so that $\left|\mu_{n}\right|\left(A_{i}\right)>\frac{\delta}{2}$, for all $1 \leq i \leq N$. Then $\left|\mu_{n}\right|\left(\cup_{i=1}^{N} A_{i}\right)>\frac{N \delta}{2}>M$, a contradiction. By passing to a subsequence, we may (and do) assume that $\sum_{k} x_{k}<\epsilon / 2$.

Partition $\mathbb{N}$ into a pairwise disjoint sequence of infinite sets $\left(M_{i}^{1}\right)$, and let $n_{1}=1$. Since $\left|\mu_{n_{1}}\right|(\Omega)<\infty$ and $\left(A_{i}\right)$ is a pairwise disjoint sequence, $\sum_{j}\left|\mu_{n_{1}}\right|\left(A_{j}\right)<\infty$. Choose $i \in \mathbb{N}$ such that

$$
\sum_{j \in M_{i}^{1}}\left|\mu_{n_{1}}\right|\left(A_{j}\right)<\epsilon / 2 .
$$

Let $N_{1}=\left\{n \in M_{i}^{1}: n>n_{1}\right\}$. Since $\lim _{n}\left|\mu_{n}\right|\left(A_{n_{1}}\right)<\epsilon / 2$ and $N_{1}$ is infinite, we can choose $n_{2} \in N_{1}$ such that $\left|\mu_{n_{2}}\right|\left(A_{n_{1}}\right)<\epsilon / 2$.

Now partition $\left\{n \in N_{1}: n>n_{1}\right\}$ into a pairwise disjoint sequence of infinite sets $\left(M_{i}^{2}\right)$. As above, choose $i \in \mathbb{N}$ so that $\sum_{j \in M_{i}^{2}}\left|\mu_{n_{2}}\right|\left(A_{j}\right)<\epsilon / 2$. Let $N_{2}=\left\{n \in M_{i}^{2}: n>\right.$ $\left.n_{2}\right\}$ and choose $n_{3} \in N_{2}$ such that $\left|\mu_{n_{3}}\right|\left(A_{n_{1}}\right)+\left|\mu_{n_{3}}\right|\left(A_{n_{2}}\right)<\epsilon / 2$.

Continuing this way we obtain a strictly increasing subsequence $\left(n_{i}\right)$ of positive integers and a sequence of infinite subsets $\left(N_{i}\right)$ of $\mathbb{N}$ so that $n_{i+1} \in N_{i}, N_{i+1} \subseteq\left\{n \in N_{i}\right.$ : $\left.n>n_{i}\right\}$,

$$
\sum_{j \in N_{i}}\left|\mu_{n_{i}}\right|\left(A_{j}\right)<\epsilon / 2
$$

and $\sum_{j=1}^{i-1}\left|\mu_{n_{i}}\right|\left(A_{n_{j}}\right)<\epsilon / 2$ 
If $j>i$, then $n_{j} \in N_{i}$, and $\sum_{j>i}\left|\mu_{n_{i}}\right|\left(A_{n_{j}}\right) \leq \sum_{j \in N_{i}}\left|\mu_{n_{i}}\right|\left(A_{j}\right)<\epsilon / 2$ for each $i$. Hence, $\sum_{j \neq i}\left|\mu_{n_{i}}\right|\left(A_{n_{j}}\right)<\epsilon$ for each $i$.

Rosenthal [22] showed that his lemma easily demonstrates that if $T: \ell_{\infty} \rightarrow X$ is an operator so that $\left\|T\left(e_{n}\right)\right\| \not \rightarrow 0$, then there is a copy of $\ell_{\infty}$ on which $T$ is an isomorphism. This result of Rosenthal and classical results of Bessaga and Pelczynski [4] are used to give a new and quick proof of the Diestel-Faires theorem.

ThEOREM 2 (Diestel-Faires theorem [6]). If $\mathcal{A}$ is an algebra, and $\mu: \mathcal{A} \rightarrow X$ is a bounded vector measure which is not strongly additive, then there is an isomorphism $T: c_{0} \rightarrow X$ and a pairwise disjoint sequence of sets $\left(A_{i}\right)$ in $\mathcal{A}$ so that $T\left(e_{n}\right)=\mu\left(A_{n}\right)$. Moreover, if $\mathcal{A}$ is a $\sigma$-algebra, the above conclusion remains true if $c_{0}$ is replaced by $\ell_{\infty}$.

Proof. Suppose that $\mathcal{A}$ is an algebra, and $\mu: \mathcal{A} \rightarrow X$ is a non-strongly additive bounded vector measure. Let $\left(A_{n}\right)$ be a sequence of pairwise disjoint sets in $\mathcal{A}$ so that $\left(\mu\left(A_{n}\right)\right) \nrightarrow \rightarrow 0$. Without loss of generality, suppose $\left\|\mu\left(A_{n}\right)\right\|>\epsilon$ for all $n$, for some $\epsilon>0$. If $x^{*} \in X^{*},\left\|x^{*}\right\| \leq 1$, then $x^{*} \mu$ is a bounded real-valued measure (since $\mu$ is bounded), thus strongly additive. Then $\sum\left|x^{*} \mu\left(A_{n}\right)\right|<\infty$, and $\sum \mu\left(A_{n}\right)$ is weakly unconditionally converging, but not unconditionally converging. Moreover, inf $\left\|\mu\left(A_{n}\right)\right\|>0$. By Lemma 3 in [4, p. 160], $\left(\mu\left(A_{n}\right)\right) \sim\left(e_{n}\right)$.

Now suppose $\mathcal{A}$ is a $\sigma$-algebra, and $\mu: \mathcal{A} \rightarrow X$ is a non-strongly additive and bounded vector measure. As above, let $\left(A_{n}\right)$ be a sequence of pairwise disjoint sets in $\mathcal{A}$ and $\epsilon>0$ so that $\left\|\mu\left(A_{n}\right)\right\|>\epsilon$ for all $n$. If $b \in \ell_{\infty}$ is a finitely valued sequence, write $b=\sum_{j=1}^{n} b_{j} \chi_{B_{j}}$, where $B_{1}, B_{2}, \ldots, B_{n}$ are pairwise disjoint sets of positive integers. Let $U$ be the dense linear subspace of $\ell_{\infty}$ consisting of finitely valued sequences, and define $T: U \rightarrow X$ by

$$
T(b)=\sum_{j=1}^{n} b_{j} \mu\left(\bigcup_{k \in B_{j}} A_{k}\right) .
$$

Note that $T$ is a bounded linear operator defined on a dense subspace of $\ell_{\infty}$, and, thus, it has a bounded extension, still denoted by $T$. Then $T: \ell_{\infty} \rightarrow X$ is a bounded linear operator and $\left\|T\left(e_{n}\right)\right\|=\left\|\mu\left(A_{n}\right)\right\|>\epsilon$ for all $n$. By Rosenthal's result [22], there is an infinite subset $M$ of $\mathbb{N}$ so that $\left.T\right|_{\ell_{\infty}(M)}$ is an isomorphic embedding. If $B_{n}=A_{n}$, $n \in M$, then $\left(B_{n}\right)$ is a pairwise disjoint sequence in $\mathcal{A}$ and $T\left(e_{n}\right)=\mu\left(B_{n}\right)$.

Theorem 2 also enables (numerous) short proofs of the measure theoretic version of the Orlicz-Pettis theorem: If $\Sigma$ is a $\sigma$-algebra and $\mu: \Sigma \rightarrow X$ is weakly countably additive, then $\mu$ is countably additive. Specifically, suppose $\left(A_{i}\right)$ is a pairwise disjoint sequence in $\Sigma, \mathcal{A}=\sigma\left(\left(A_{i}\right)\right)$, and $Y=\left[\mu\left(A_{i}\right): i \geq 1\right]$. Weak countable additivity of $\mu$ and separability of $Y$ ensure that $\mu: \mathcal{A} \rightarrow Y$ is strongly additive. It is straightforward to check that a strongly additive and weakly countably additive measure is countably additive, i.e. $\mu\left(\cup_{i=1}^{\infty} A_{i}\right)=\sum_{i=1}^{\infty} \mu\left(A_{i}\right)$.

The next result links unconditional convergence in the strong operator topology and the presence of isomorphic copies of $\ell_{\infty}$ in $L(E, F)$.

THEOREM 3. (i)If $\ell_{1} \stackrel{s}{\rightarrow} E$ and $\left(T_{n}\right)$ is a sequence of operators in $L(E, F)$ so that $\sum T_{n}(x)$ converges unconditionally for all $x \in E$, then $\sum T_{n}^{*}\left(y^{*}\right)$ converges unconditionally for all $y^{*} \in F^{*}$. 
(ii) If $\ell_{1} \stackrel{c}{\hookrightarrow} E$, then there is a sequence $\left(T_{n}\right)$ of operators in $L(E, F)$ so that $\sum T_{n}(x)$ is unconditionally convergent for all $x \in E$ and $\sum T_{n}^{*}\left(y^{*}\right)$ is not unconditionally convergent for some $y^{*} \in F^{*}$.

(iii) If $\left(T_{n}\right)$ is a sequence in $L(E, F)$ so that $\sum T_{n}(x)$ is unconditionally convergent for all $x \in E$ and $\sum T_{n}^{*}\left(y^{*}\right)$ fails to converge for some $y^{*} \in F^{*}$, then $\ell_{\infty} \hookrightarrow L(E, F)$.

Proof. (i) Suppose $\ell_{1} \stackrel{c}{\rightarrow} E$, and let $\left(T_{n}\right)$ be a sequence in $L(E, F)$ such that $\sum T_{n}(x)$ converges unconditionally for all $x \in E$. If $y^{*} \in F^{*}$, then $\sum T_{n}^{*}\left(y^{*}\right)$ converges unconditionally in the $w^{*}$ - topology of $E^{*}$. By the Bessaga-Pelczyinski theorem [4] (or [16, Proposition 3]), $\sum_{c} T_{n}^{*}\left(y^{*}\right)$ converges unconditionally for all $y^{*} \in F^{*}$.

(ii) Suppose $\ell_{1} \stackrel{c}{\hookrightarrow} E$. Let $P: E \rightarrow \ell_{1}$ be a projection and let $y$ be a normalised element of $F$. Define $T_{n}: E \rightarrow F$ by $T_{n}(x)=\left\langle P(x), e_{n}\right\rangle y=e_{n} P(x) y$. Note that $\sum T_{n}(x)$ is absolutely summable, and thus unconditionally converging for all $x \in E$. If $y^{*} \in F^{*}$, $\sum T_{n}^{*}\left(y^{*}\right)=\sum y^{*}(y) e_{n} P$. However, $\left\|e_{n} P\right\| \not \rightarrow 0$, and thus $\sum T_{n}^{*}\left(y^{*}\right)$ does not converge unconditionally if $y^{*}(y) \neq 0$.

(iii) Suppose $\sum T_{n}(x)$ converges unconditionally for all $x \in E$ and $\sum T_{n}^{*}\left(y^{*}\right)$ fails to converge for some $y^{*} \in F^{*}$. Let $\epsilon>0$ and $\left(p_{i}, q_{i}\right)$ be a sequence of intertwining pairs of positive integers so that $\left\|\sum_{n=p_{i}}^{q_{i}} T_{n}^{*}\left(y^{*}\right)\right\| \geq \epsilon$ for each $i$. Let $L_{i}=\sum_{n=p_{i}}^{q_{i}} T_{n}$. Certainly, $\left\|L_{i}\right\| \not \rightarrow 0$, and $\sum L_{i}(x)$ is unconditionally convergent for each $x \in E$. Define $\mu: \mathcal{P} \rightarrow L(E, F)$ by $\mu(A)=\sum_{i \in A} L_{i}($ sot $)$. Then $\mu$ is bounded, finitely additive and not strongly additive $(\mu(\{i\}) \not \nrightarrow 0)$. Apply the Diestel-Faires theorem to conclude that $\ell_{\infty} \hookrightarrow L(E, F)$.

REMARK. If $\ell_{\infty} \hookrightarrow L(E, F)$, then $\ell_{\infty} \hookrightarrow L\left(F^{*}, E^{*}\right)$. To see this, let $U: \ell_{\infty} \rightarrow$ $L(E, F)$ be an isomorphism and consider $V: \ell_{\infty} \rightarrow L\left(F^{*}, E^{*}\right), V(b)=U(b)^{*}$. If $E$ and $F$ are infinite dimensional, the question of whether $\ell_{\infty} \hookrightarrow L(E, F)$ when $\ell_{\infty} \hookrightarrow L\left(F^{*}, E^{*}\right)$ is more delicate and will be discussed later in this paper.

Suppose $p: \mathcal{P} \rightarrow \mathbb{R}$ is a non-negative, finitely additive and real-valued set function defined on the power class of the natural numbers, e.g. $p$ is the total variation of an element in $b a(\mathcal{P})$. Suppose that $p(\{n\})=0$ for each $n$. Let $\left\{A_{\alpha}: \alpha \in D\right\}$ be an uncountable collection of infinite subsets of the natural numbers so that $A_{\alpha} \cap A_{\beta}$ is finite if $\alpha \neq \beta$, i.e. $A_{\alpha}$ and $A_{\beta}$ are almost disjoint. Then $p\left(A_{\alpha}\right)=0$ for uncountably many $\alpha$. If not, there is a $\delta>0$ so that $\left\{\alpha: p\left(A_{\alpha}\right)>\delta\right\}$ is uncountable. Since $p\left(A_{\alpha} \cup\right.$ $\left.A_{\beta}\right)=p\left(A_{\alpha}\right)+p\left(A_{\beta}\right)$ for $\alpha \neq \beta$, we contradict the assumption that $p(\mathbb{N})$ is real. Now suppose $\mu: \mathcal{P} \rightarrow X$ is bounded and finitely additive, $\mu(\{n\})=0$ for all $n$ and $\left(x_{n}^{*}\right)$ is a sequence of functionals in $X^{*}$ which separates the points in $\mu(\mathcal{P})$. If one assumes that there are uncountably many $\alpha$ for which $|\mu|\left(A_{\alpha}\right) \neq 0$, then there is an $n$ so that $\left\{\alpha:\left|x_{n}^{*} \mu\right|\left(A_{\alpha}\right)>0\right\}$ is uncountable. Let $p=\left|x_{n}^{*} \mu\right|$, and note the contradiction above.

LEMMA 4. If $\mu: \mathcal{P} \rightarrow X$ is bounded and finitely additive, $\mu(\{n\})=0$ for all $n$, and there are countably many functionals in $X^{*}$ separating the points in $\mu(\mathcal{P})$, then there is an uncountable collection $\left\{A_{\alpha}: \alpha \in D\right\}$ of almost disjoint infinite subsets of $\mathbb{N}$ so that if $\alpha \in D$ and $B \subseteq A_{\alpha}$, then $\mu(B)=0$.

We remark that if $E$ is separable and $F$ is the dual of a separable space, then there are countably many functionals separating the points of $L(E, F)$. Lemma 4 furnishes an almost immediate proof of Drewnowski's result, which was highlighted in the Introduction. Specifically, suppose $\mu: \Sigma \rightarrow X$ is a strongly additive measure and $\lambda$ is a finitely additive non-negative real-valued measure so that $\mu \ll \lambda$. Let $\left(A_{i}\right)$ be a pairwise 
disjoint sequence of sets in $\Sigma$ and identify $\sigma\left(\left(A_{i}\right)\right)$ with $\mathcal{P}$. Define $v(S)=\sum_{i \in S} \lambda(\{i\})$, for $S \subseteq \mathbb{N}$. Note that $v: \mathcal{P} \rightarrow \mathbb{R}$ is countably additive and $v(\{i\})=\lambda(\{i\})$ for each $i$. Lemma 4 produces an infinite subset $M$ of $\mathbb{N}$ so that $v(S)=\lambda(S)$ for all $S \subseteq M$. If $B_{i}=A_{i}, i \in M$, then $\left(B_{i}\right)$ is a subsequence of $\left(A_{i}\right)$, and $\lambda$ is countably additive on $\sigma\left(\left(B_{i}\right)\right)$. Since $\mu$ is $\lambda$-continuous, $\mu$ is countably additive on $\sigma\left(\left(B_{i}\right)\right)$.

Lemma 4 also plays an important role in the proofs of the next 11 results, each of which deals with the structure of spaces of operators. Theorem 5 presents an alternate and shorter proof of the main result in [7], Theorem 6 uses the family of projections associated with an unconditional basic sequence together with auxiliary maps to show that certain subspaces of $L(X, Y)$ are not complemented, Corollaries 7-13 extend results in several papers listed in the bibliography and Theorem 14 explicitly formalises a phenomenon present in preceding results.

The following isometries [23] help to establish the context of Theorem 5.

(1) $L_{w^{*}}\left(X^{*}, Y\right) \simeq L_{w^{*}}\left(Y^{*}, X\right), K_{w^{*}}\left(X^{*}, Y\right) \simeq K_{w^{*}}\left(Y^{*}, X\right)\left(T \rightarrow T^{*}\right)$

(2) $W(X, Y) \simeq L_{w^{*}}\left(X^{* *}, Y\right)$ and $K(X, Y) \simeq K_{w^{*}}\left(X^{* *}, Y\right)\left(T \rightarrow T^{* *}\right)$.

TheOREM 5. (Drewnowski, [7]). $\ell_{\infty} \hookrightarrow K_{w^{*}}\left(X^{*}, Y\right)$ iff $\ell_{\infty} \hookrightarrow X$ or $\ell_{\infty} \hookrightarrow Y$.

Proof. Suppose $\ell_{\infty} \hookrightarrow X$ and $\ell_{\infty} \hookrightarrow Y$. Let $T: \ell_{\infty} \rightarrow K_{w^{*}}\left(X^{*}, Y\right)$ be an isomorphism. For $x^{*} \in X^{*}, y^{*} \in Y^{*}$, define $T_{x^{*}}: \ell_{\infty} \rightarrow Y$ and $A_{y^{*}}: \ell_{\infty} \rightarrow X$ by $T_{x^{*}}(b)=T(b)\left(x^{*}\right)$ and $A_{y^{*}}(b)=T(b)^{*}\left(y^{*}\right)$. Note that since $\ell_{\infty} \hookrightarrow X$ and $\ell_{\infty} \hookrightarrow Y$, the operators $T_{x^{*}}: \ell_{\infty} \rightarrow Y$ and $A_{y^{*}}: \ell_{\infty} \rightarrow X$ are weakly compact ([22], [6, Chapter $\mathrm{VI}]$ ), and thus unconditionally converging. Let $T_{i}=T\left(e_{i}\right)$ for each $i$, and let

$$
S(b)=\sum b_{i} T_{i} \quad(s o t)
$$

for each $b=\left(b_{i}\right) \in \ell_{\infty}$. By the uniform boundedness principle (or the Pietsch $\epsilon$-norm [20, Section 1.2]), we obtain that $S(b)$ is a bounded linear operator. Further, $S(b) \in$ $L_{w^{*}}\left(X^{*}, Y\right)$.

Let $R$ be the rational span of $\left\{T_{i}: i \geq 1\right\}$. Certainly, $R$ is countable. Further, if $L \in R$, then $L$ is compact. Thus, there is a separable closed linear subspace $H$ of $X^{*}$ so that $S(b)\left(B_{X^{*}}\right) \subseteq \overline{S(b)\left(B_{H}\right)} \subseteq\left[T_{i}(H): i \geq 1\right]$. Now we observe that $\left[T_{i}(H): i \geq 1\right]$ is a separable subspace of $Y$. Thus, there is a continuous linear transformation $W: Y \rightarrow$ $\ell_{\infty}$ so that $\left.W\right|_{\left[T_{i}(H): i \geq 1\right]}$ is an isometry.

Let $\mu(A)=\left.W T\left(\chi_{A}\right)\right|_{H}$ and $v(A)=W\left(\left.\sum_{i \in A} T_{i}\right|_{H}\right)=\left.W S\left(\chi_{A}\right)\right|_{H}$, for $A \subseteq \mathbb{N}$. Then $\mu, v: \mathcal{P} \rightarrow L\left(H, \ell_{\infty}\right)$ are bounded and finitely additive measures, and $\mu(\{n\})=v(\{n\})$ for all $n$. Thus, by Lemma 4 , there is an infinite subset $M$ of $\mathbb{N}$ so that $\mu(A)=v(A)$ for all $A \subseteq M$. Consequently, $W\left(\left.\sum_{i \in A} T_{i}\right|_{H}\right)$ is compact for all $A \subseteq M$. Since $W$ is an isometry on $\left[T_{i}(H): i \geq 1\right],\left.\sum_{i \in A} T_{i}\right|_{H}$ is compact. Then $\sum_{i \in A} T_{i}$ is compact for all $A \subseteq M$ (by the construction of $H$ ).

For $x^{*} \in X^{*}$ and $b \in \ell_{\infty}(M), S(b)\left(x^{*}\right)=\sum_{i \in M} b_{i} T_{i}\left(x^{*}\right)$. Then $S(b)=\sum_{i \in M} b_{i} T_{i}$ (weakly) in $K_{w^{*}}\left(X^{*}, Y\right)$ [14], and by the Orlicz-Pettis theorem, $S(b)=\sum_{i \in M} b_{i} T_{i}$ (norm) in $K_{w^{*}}\left(X^{*}, Y\right)$, for each $b \in \ell_{\infty}(M)$. Hence, $\left\|T_{i}\right\| \rightarrow 0, i \in M$, a contradiction since $T$ is an isomorphism.

Conversely, suppose $\ell_{\infty} \hookrightarrow X\left(\ell_{\infty} \hookrightarrow Y\right)$. Since $X$ and $Y$ embed in the rank one operators from $X^{*}$ to $Y, \ell_{\infty} \hookrightarrow K_{w^{*}}\left(X^{*}, Y\right)$.

THEOREM 6. (i) Let $X$ and $Y$ be Banach spaces with the following properties:

There exists a Banach space $G$ with a semi-normalised unconditional basis $\left(g_{i}\right)$ and coefficient functionals $\left(g_{i}^{*}\right)$, and operators $R: G \rightarrow Y$ and $S: X \rightarrow G$ such that $\left(R\left(g_{i}\right)\right)$ is 
a semi-normalised basic sequence in $Y$ and $\left(S^{*}\left(g_{i}^{*}\right)\right)$ has no norm convergent subsequence. If $\left(P_{A}\right)$ is the family of projections associated to $\left(g_{i}\right)$ and $T: L(X, Y) \rightarrow K(X, Y)$ is an operator, then there is an $N \in \mathbb{N}$ so that

$$
T R P_{\{n\}} S \neq R P_{\{n\}} S,
$$

for $n>N$. Thus, $K(X, Y)$ is not complemented in $L(X, Y)$.

(ii) Let $X$ and $Y$ be Banach spaces with the following properties:

There exists a Banach space $G$ with a semi-normalised unconditional basis $\left(g_{i}\right)$ and coefficient functionals $\left(g_{i}^{*}\right)$, and operators $R: G \rightarrow Y$ and $S: X \rightarrow G$ such that $\left(R\left(g_{i}\right)\right)$ is a semi-normalised basic sequence in $Y$ and $\left(S^{*}\left(g_{i}^{*}\right)\right)$ has no weakly convergent subsequence. If $\left(P_{A}\right)$ is the family of projections associated to $\left(g_{i}\right)$ and $T: L(X, Y) \rightarrow W(X, Y)$ is an operator, then there is an $N \in \mathbb{N}$ so that

$$
T R P_{\{n\}} S \neq R P_{\{n\}} S,
$$

for $n>N$. Thus, $W(X, Y)$ is not complemented in $L(X, Y)$.

(iii) Let $X$ and $Y$ be Banach spaces with the following properties:

There exists a Banach space $G$ with a semi-normalised unconditional basis $\left(g_{i}\right)$ and coefficient functionals $\left(g_{i}^{*}\right)$, and operators $R: G \rightarrow Y$ and $S: X \rightarrow G$ such that $R$ is weakly compact, $\left(R\left(g_{i}\right)\right)$ is a semi-normalised basic sequence in $Y$ and $\left(S^{*}\left(g_{i}^{*}\right)\right)$ has no norm convergent subsequence. If $\left(P_{A}\right)$ is the family of projections associated to $\left(g_{i}\right)$ and $T: W(X, Y) \rightarrow K(X, Y)$ is an operator, then there is an $N \in \mathbb{N}$ so that

$$
T R P_{\{n\}} S \neq R P_{\{n\}} S,
$$

for $n>N$. Thus, $K(X, Y)$ is not complemented in $W(X, Y)$.

Proof. (i) Let $\left(P_{A}\right)$ be the family of projections associated with $\left(g_{n}\right), R$ and $S$ as in the hypothesis, and set $\mu(A)=R P_{A} S, A \subseteq \mathbb{N}$. Let $X_{0}$ be a separable subspace of $X$ such that $\left\|x^{*}\right\|=\left\|\left.x^{*}\right|_{X_{0}}\right\|$ for all $x^{*} \in\left[S^{*}\left(g_{n}^{*}\right): n \geq 1\right]$. Let $\left(y_{n}^{*}\right)$ be the sequence of biorthogonal coefficients corresponding to $\left(R\left(g_{n}\right)\right)$, and $\left(f_{n}^{*}\right)$ be the sequence of Hahn-Banach extensions to $Y^{*}$. Note that $\left.\mu(A)\right|_{X_{0}}$ is compact iff $A$ is finite. Indeed, $\left(\mu(A)^{*}\left(f_{n}^{*}\right)\right)=\left(S^{*}\left(g_{n}^{*}\right)\right)_{n \in A}$, which is relatively compact iff $A$ is finite.

Now suppose $T: L(X, Y) \rightarrow K(X, Y)$ is an operator and $B=\{n \in \mathbb{N}: T \mu(\{n\})=$ $\mu(\{n\})\}$ is an infinite set. Let $J: Y \rightarrow \ell_{\infty}$ be an operator which is an isometry on $\left[R\left(g_{n}\right)\right.$ : $n \geq 1$ ]. Identify $\mathcal{P}$ with $\mathcal{P}(B)$ in the obvious way, and define $v: \mathcal{P}(B) \rightarrow L\left(X_{0}, \ell_{\infty}\right)$ by $\nu(A)=\left.(J T \mu(A)-J \mu(A))\right|_{X_{0}}, A \subseteq B$. Apply Lemma 4 to obtain an infinite subset $M$ of $B$ so that $J T \mu(M)=J \mu(M)$ on $X_{0}$. Since $J$ is an isometry on $\left[R\left(g_{n}\right): n \geq 1\right]$ and $\left.J T \mu(M)\right|_{X_{0}}$ is compact, $\left.\mu(M)\right|_{X_{0}}$ is also compact, a contradiction. Therefore there does not exist a projection $P: L(X, Y) \rightarrow K(X, Y)$.

(ii) The proof is essentially the same as the proof of (i), replacing 'relatively compact' with 'relatively weakly compact'.

(iii) The proof is similar to the proof of (i), defining $\mu: \mathcal{P} \rightarrow W(X, Y)$ by $\mu(A)=R P_{A} S, A \subseteq \mathbb{N}$, and $v: \mathcal{P}(B) \rightarrow W\left(X_{0}, \ell_{\infty}\right)$ by $v(A)=\left.(J T \mu(A)-J \mu(A))\right|_{X_{0}}$, $A \subseteq B$.

REMARK. If one assumes that $\left\{S^{*}\left(g_{i}^{*}\right): i \in \mathbb{N}\right\}$ is not relatively compact, then one may pass to a subsequence $\left(g_{n_{i}}\right)$ and obtain the same conclusion as above.

COROLlary 7 ([9], [19]). If $X$ has a semi-normalised unconditional basis $\left(x_{n}\right)$, $\left(P_{A}\right)$ is the family of projections associated with $\left(x_{n}\right)$ and $T: L(X, X) \rightarrow K(X, X)$ is 
an operator, then there is an $N \in \mathbb{N}$ so that $T P_{\{n\}} \neq P_{\{n\}}$, for $n>N$. Thus, $K(X, X)$ is not complemented in $L(X, X)$.

Proof. Let $G=Y=X$, and $R=S$ be the identity on $X$. Apply Theorem 6 .

Corollary 8 (Lemma 3, [16]). If $\ell_{1}$ is complemented in $X$ and $Y$ is infinite dimensional, then $K(X, Y)$ is not complemented in $L(X, Y)$.

Proof. Let $P$ be a projection from $X$ to $\ell_{1}$. Let $G=\ell_{1}$. Since $P$ is a projection, $P^{*}$ is an isomorphism. Therefore $\left(P^{*}\left(e_{n}\right)\right)$ has no norm convergent subsequence. Let $\left(y_{n}\right)$ be a normalised basic sequence in $Y$. Define $R: \ell_{1} \rightarrow Y$ by $R(b)=\sum b_{n} y_{n}, b=\left(b_{n}\right) \in \ell_{1}$. Then $\left(R\left(e_{n}^{*}\right)\right)=\left(y_{n}\right)$ is basic and normalised. Apply Theorem 6 .

If each of $\left(x_{n}\right)$ and $\left(y_{n}\right)$ is a basic sequence, one says that $\left(x_{n}\right)$ dominates $\left(y_{n}\right)$ and writes $\left(x_{n}\right) \succ\left(y_{n}\right)$ provided that $\sum \alpha_{n} y_{n}$ converges whenever $\sum \alpha_{n} x_{n}$ converges. Note that if $\left(y_{n}\right)$ is any semi-normalised basic sequence, then $\left(e_{n}^{*}\right) \succ\left(y_{n}\right)$. Singer [24, p. 68] shows that if $\left(x_{n}\right)$ and $\left(y_{n}\right)$ are semi-normalised basic sequences and $\left(x_{n}\right) \succ\left(y_{n}\right)$, then there is an operator $T:\left[x_{n}\right] \rightarrow\left[y_{n}\right]$ so that $T\left(x_{n}\right)=y_{n}$ for each $n$. Theorem 6 and the proof of Corollary 8 immediately produce the following generalisation of Lemma 3 of [16]: If $\left(x_{n}\right)_{n=1}^{\infty}$ is an unconditional semi-normalised basic sequence, $\left[x_{n}\right]$ is complemented in $X,\left(y_{n}\right)_{n=1}^{\infty}$ is a semi-normalised basic sequence in $Y$ and $\left(x_{n}\right) \succ\left(y_{n}\right)$, then $K(X, Y)$ is not complemented in $L(X, Y)$.

Corollary 9 (11, Corollary 4). If $c_{0} \hookrightarrow Y$ and $X$ is infinite dimensional, then $K(X, Y)$ is not complemented in $L(X, Y)$.

Proof. Let $\left(x_{n}^{*}\right)$ be a $w^{*}$-null sequence of norm one elements in $X^{*}$ (JosefsonNissenzweig theorem). Define $S: X \rightarrow c_{0}$ by $S(x)=\left(x_{n}^{*}(x)\right)$. Note that $\left(S^{*}\left(e_{n}^{*}\right)\right)=\left(x_{n}^{*}\right)$ has no norm convergent subsequence. Let $G=c_{0}$ and $R: c_{0} \rightarrow Y$ be an embedding. Apply Theorem 6.

Corollary 10 ([8, Theorems 2 and 3]; [2, Theorem 4]). If $c_{0} \hookrightarrow Y$ and $X^{*}$ contains a $w^{*}$-null sequence $\left(x_{n}^{*}\right)$, which is not weakly null, then $W(X, Y)$ is not complemented in $L(X, Y)$.

Proof. Assume without loss of generality that $\left(x_{n}^{*}\right)$ has no weakly convergent subsequence. Define $S: X \rightarrow c_{0}$ by $S(x)=\left(x_{n}^{*}(x)\right)$. Then $\left(S^{*}\left(e_{n}^{*}\right)\right)=\left(x_{n}^{*}\right)$ has no weakly convergent subsequence. Let $G=c_{0}$ and $R: c_{0} \rightarrow Y$ be an embedding. Apply Theorem 6.

Corollary 11 (Theorem 2, [9, 15]). If $c_{0} \hookrightarrow K(X, Y)$, then $K(X, Y)$ is not complemented in $L(X, Y)$.

Proof. By corollaries 8 and 9 we can suppose without loss of generality that $c_{0} \hookrightarrow$ $X^{*}$ and $c_{0} \hookrightarrow Y$. Suppose $c_{0} \hookrightarrow K(X, Y)$ and let $\left(T_{n}\right)$ be a copy of $\left(e_{n}\right)$ in $K(X, Y)$. Then $\sum T_{n}(x)$ converges unconditionally for each $x \in X$. Define $\mu: \mathcal{P} \rightarrow L(X, Y)$ by $\mu(A)(x)=\sum_{n \in A} T_{n}(x), x \in X, A \subseteq \mathbb{N}$. If $R: L(X, Y) \rightarrow K(X, Y)$ is an operator, then $R T_{n}=T_{n}$ for only finitely many $n$; in fact, $\left\|R T_{n}\right\| \rightarrow 0$. Otherwise, apply Theorem 2 to the measure $R \mu$ and obtain $\ell_{\infty} \hookrightarrow K(X, Y)$. Theorem 5 and the present hypotheses eliminate this possibility.

Corollary 12 (10, Lemma 3). If $\ell_{1}$ is complemented in $X$ and $Y$ does not have the Schur property, then $K(X, Y)$ is not complemented in $W(X, Y)$. 
Proof. Let $P$ be a projection from $X$ to $\ell_{1}$. Let $G=\ell_{1}$. The proof of Corollary 8 shows that $\left(P^{*}\left(e_{n}\right)\right)$ has no norm convergent subsequence. Let $\left(y_{n}\right)$ be a normalised weakly null basic sequence in $Y$. Define $R: \ell_{1} \rightarrow Y$ by $R(b)=\sum b_{n} y_{n}, b=\left(b_{n}\right) \in \ell_{1}$. Since $\left(R\left(e_{n}^{*}\right)\right)=\left(y_{n}\right)$ is weakly null, $R$ is weakly compact. Apply Theorem 6 .

Corollary 13 ([2, Theorem 3]). Suppose that $X$ has a semi-normalised unconditional basis $\left(x_{n}\right),\left(P_{A}\right)$ is the family of projections associated with $\left(x_{n}\right)$ and $S: X \rightarrow Y$ is an operator so that no subsequence of $\left(S\left(x_{n}\right)\right)$ converges weakly. If $T: L(X, Y) \rightarrow W(X, Y)$ is an operator, then there is an $N \in \mathbb{N}$ so that $T S P_{\{n\}} \neq S P_{\{n\}}$, for $n>N$. Thus, $W(X, Y)$ is not complemented in $L(X, Y)$.

Consequently, if $\ell_{1}$ is complemented in $X$ and $W(X, Y) \neq L(X, Y)$, then $W(X, Y)$ is not complemented in $L(X, Y)$.

Proof. Let $\left(P_{A}\right)$ be the family of associated projections, $S: X \rightarrow Y$ be as in the hypothesis and set $\mu(A)=S P_{A}, A \subseteq \mathbb{N}$. Note that $\mu(A)$ is weakly compact iff $A$ is finite. Let $J: Y \rightarrow \ell_{\infty}$ be an operator, which is an isometry on $\left[S\left(x_{n}\right): n \geq 1\right]$. Now, suppose that $T: L(X, Y) \rightarrow W(X, Y)$ is an operator and $B=\{n: T \mu(\{n\})=\mu(\{n\})\}$ is an infinite set. Identify $\mathcal{P}$ with $\mathcal{P}(B)$ in the obvious way, and define $v: \mathcal{P}(B) \rightarrow L\left(X, \ell_{\infty}\right)$ by $v(A)=J T \mu(A)-J \mu(A), A \subseteq B$. Apply Lemma 4 to obtain an infinite subset $M$ of $B$ so that $J T \mu(M)=J \mu(M)$. Since $J$ is an isometry on $\left[S\left(x_{n}\right): n \geq 1\right]$ and $J T \mu(M)$ is weakly compact, $\mu(M)$ is weakly compact, a contradiction. Therefore there does not exist a projection $P: L(X, Y) \rightarrow W(X, Y)$.

Now, suppose that $\ell_{1}$ is complemented in $X$ and $W(X, Y) \neq L(X, Y)$. If $W(X, Y)$ is complemented in $L(X, Y)$, then $W\left(\ell_{1}, Y\right)$ is complemented in $L\left(\ell_{1}, Y\right)$. Let $\left(y_{n}\right)$ be a bounded sequence in $Y$ with no weakly convergent subsequence and define $S$ : $\ell_{1} \rightarrow Y$ by $S(x)=\sum x_{n} y_{n}, x=\left(x_{n}\right) \in \ell_{1}$. The preceding argument shows that there is a sequence of rank one operators $\left(e_{n}^{*} \otimes y_{n}\right)$ so that any operator $T: L\left(\ell_{1}, Y\right) \rightarrow W\left(\ell_{1}, Y\right)$ fixes at most finitely many terms of this sequence.

The measure $\mu$ in the first paragraph of the proof of Theorem 6 has the property that $\left.\mu(A)\right|_{X_{0}}$ is compact iff $A$ is finite. The next result explicitly demonstrates the significance of such a measure.

Theorem 14. Suppose that $X$ is separable and $Y$ embeds in the dual of a separable space. Suppose further that $U$ is an infinite set and $\mu: \mathcal{P}(U) \rightarrow L(X, Y)$ is a bounded and finitely additive measure so that $\mu(A) \in K(X, Y)$ iff $A$ is finite. If $T: L(X, Y) \rightarrow$ $K(X, Y)$ is an operator and $\left(u_{n}\right)$ is an infinite sequence of distinct points in $U$, then there is an $N \in \mathbb{N}$ so that $T \mu\left(\left\{u_{n}\right\}\right) \neq \mu\left(\left\{u_{n}\right\}\right)$, for $n>N$. Thus, $K(X, Y)$ is not complemented in $L(X, Y)$.

Proof. Note first that there are countably many continuous linear functionals separating the points of $L(X, Y)$. Suppose there exists an operator $T: L(X, Y) \rightarrow$ $K(X, Y)$ so that $T \mu\left(\left\{u_{n}\right\}\right)=\mu\left(\left\{u_{n}\right\}\right)$ for infinitely many $n$. Identify the power class of the natural numbers with the power class of the subsequence satisfying the preceding equality, and define $v: \mathcal{P} \rightarrow L(X, Y)$ by $v(B)=T \mu(B)-\mu(B), B \in \mathcal{P}$. Lemma 4 produces an infinite subset $M$ of $U$ so that $v(B)=0$ for all $B \subseteq M$. Therefore $T \mu(M)=\mu(M)$, a contradiction since $\mu(M)$ is not compact. Thus, there does not exist a projection $P: L(X, Y) \rightarrow K(X, Y)$.

We now address the question raised in the remark following the proof of Theorem 3. If $\ell_{\infty}$ embeds in $L\left(F^{*}, E^{*}\right)$, does $\ell_{\infty}$ embed in $L(E, F)$ ? Suppose $1<p<2<q<\infty$, 
and consider $L\left(\ell_{q}, \ell_{p}\right)$. Since every operator $T: \ell_{q} \rightarrow \ell_{p}$ is compact [21] and $\ell_{\infty}$ embeds in neither $\left(\ell_{q}\right)^{*}$ nor $\ell_{p}$, Theorem 5 shows that $\ell_{\infty} \hookrightarrow L\left(\ell_{q}, \ell_{p}\right)$.

Now let $G=\ell_{2}, X=\ell_{p}, Y=\ell_{q}, R: G \rightarrow Y$ be the natural inclusion, and let $S: G^{*} \rightarrow X^{*}$ be the natural inclusion map. A direct application of results in [9] and [12] shows that $c_{0} \hookrightarrow K\left(\ell_{p}, \ell_{q}\right)$, and the main result in [18] shows that $\ell_{\infty} \hookrightarrow L\left(\ell_{p}, \ell_{q}\right)$. We also observe that $c_{0} \hookrightarrow K\left(\ell_{q}, \ell_{p}\right)([13$, Theorem 9], [10, Theorem 4]).

As observed in the concluding paragraph of the proof of Theorem 5 (see also [16]), if $\ell_{\infty} \hookrightarrow X^{*}$, then $\ell_{\infty} \hookrightarrow K(X, Y)$. In fact, if $T: \ell_{\infty} \rightarrow X^{*}$ is an isomorphism, $y$ is any non-zero vector in $Y$, and $\left(T\left(e_{n}\right) \otimes y\right)(x)=T\left(e_{n}\right)(x) y$, then $\left\|T\left(e_{n}\right) \otimes y\right\| \not \rightarrow 0$, and $\sum\left(T\left(e_{n}\right) \otimes y\right)(x)$ is unconditionally convergent for all $x \in X$. Moreover, the proofs of part (iii) of Theorem 3, Theorem 3.1 in [14], Theorem 2.4 in [3] and Theorem 1 in [11] explicitly suggest the equivalence of (a) the embeddability of $\ell_{\infty}$ in $L(X, Y)$ and (b) the existence of a sequence $\left(T_{n}\right)$ in $L(X, Y)$ so that $\left\|T_{n}\right\| \not \rightarrow 0$ and $\sum T_{n}(x)$ converges unconditionally in $Y$ for each $x \in X$.

A very simple example shows that (a) does not imply (b) in general. Specifically, $\ell_{\infty} \hookrightarrow L\left(\mathbb{R}, \ell_{\infty}\right)$, and the strong operator topology in $L\left(\mathbb{R}, \ell_{\infty}\right)$ is the norm topology on $\ell_{\infty}$. However, we do have the following result.

THEOREM 15. Suppose that $X$ is infinite dimensional.

(i) If $c_{0} \hookrightarrow Y$ or $c_{0} \hookrightarrow X^{*}$, then there is a sequence $\left(T_{n}\right)$ in $K(X, Y)$ so that $\left\|T_{n}\right\| \nrightarrow 0$ and $\sum T_{n}(x)$ is unconditionally convergent for all $x \in X$.

(ii) If $c_{0} \hookrightarrow L(X, Y), c_{0} \hookrightarrow Y$, and $c_{0} \hookrightarrow X^{*}$, then there is a sequence $\left(T_{n}\right)$ in $L(X, Y)$ so that $\sum T_{n}(x)$ and $\sum T_{n}^{*}\left(y^{*}\right)$ are unconditionally convergent for all $x \in X$ and $y^{*} \in Y^{*}$, and $\left\|T_{n}\right\| \not \rightarrow 0$.

(iii) If $\ell_{\infty} \hookrightarrow L(X, Y), \ell_{\infty} \hookrightarrow Y$, and $\ell_{\infty} \hookrightarrow X^{*}$, then there is a sequence $\left(T_{n}\right)$ in $L(X, Y)$ so that $\sum T_{n}(x)$ and $\sum T_{n}^{*}\left(y^{*}\right)$ are unconditionally convergent for all $x \in X$ and $y^{*} \in Y^{*}$, and $\left\|T_{n}\right\| \not \supset 0$.

(iv) If $\left(T_{n}\right)$ is a sequence in $L(X, Y)$ so that $\left\|T_{n}\right\| \nrightarrow \rightarrow 0$ and $\sum T_{n}(x)$ is unconditionally convergent for all $x \in X$, then $\ell_{\infty} \hookrightarrow L(X, Y)$.

Proof. (i) Suppose that $c_{0} \hookrightarrow Y$ and let $\left(y_{n}\right)$ be a copy of $\left(e_{n}\right)$ in $Y$. Use the JosefsonNissenzweig theorem [5, Ch. XII], and choose a normalised $w^{*}-$ null sequence $\left(x_{n}^{*}\right)$ in $X^{*}$. Let $T_{n}: X \rightarrow Y, T_{n}=x_{n}^{*} \otimes y_{n}$; i.e. $T_{n}(x)=x_{n}^{*}(x) y_{n}$. Then $\sum T_{n}(x)$ converges unconditionally and $\left\|T_{n}\right\|=\left\|y_{n}\right\| \not \rightarrow 0$.

If $c_{0} \hookrightarrow X^{*}$, the discussion preceding this theorem demonstrates the existence of a sequence of operators that has the desired properties.

(ii) Next, suppose that $c_{0} \hookrightarrow Y$ and $c_{0} \hookrightarrow X^{*}$. Let $T: c_{0} \rightarrow L(X, Y)$ be an isomorphism, and let $T_{i}=T\left(e_{i}\right)$. Then $\left\|T_{i}\right\| \not \rightarrow 0$ and $\sum T_{i}(x)$ is weakly unconditionally convergent, and thus unconditionally converging for all $x \in X$ [4]. Further, in this case, $\sum T_{i}^{*}\left(y^{*}\right)$ is unconditionally convergent for all $y^{*} \in Y^{*}$.

(iii) Suppose that $\ell_{\infty} \hookrightarrow Y$ and $\ell_{\infty} \hookrightarrow X$. Let $T: \ell_{\infty} \rightarrow L(X, Y)$ be an isomorphism and $T_{i}=T\left(e_{i}\right)$. Note that $\left\|T_{i}\right\| \not \rightarrow 0$. For $x \in X$ and $y^{*} \in Y^{*}$, define $T_{x}: \ell_{\infty} \rightarrow Y$ by $T_{x}(b)=T(b)(x)$ and $T_{y^{*}}: \ell_{\infty} \rightarrow X^{*}$ by $T_{y^{*}}(b)=T(b)^{*}\left(y^{*}\right), b \in \ell_{\infty}$. Since $\ell_{\infty} \hookrightarrow Y, T_{x}$ is weakly compact, and thus $\sum T_{x}\left(e_{i}\right)=\sum T_{i}(x)$ is unconditionally converging for all $x \in X$ [16, Proposition 1]. Similarly, since $\ell_{\infty} \hookrightarrow X^{*}, T_{y^{*}}$ is weakly compact and $\sum T_{y^{*}}\left(e_{i}\right)=\sum T_{i}^{*}\left(y^{*}\right)$ is unconditionally convergent for all $y^{*} \in Y^{*}$.

(iv) See the proof of (iii) in Theorem 3.

REMARK. If $X$ is infinite dimensional, Theorem 15 shows that $\ell_{\infty} \hookrightarrow L(X, Y)$ precisely when there exists a sequence $\left(T_{n}\right)$ in $L(X, Y)$ so that $\left\|T_{n}\right\| \not \rightarrow 0$ and $\sum T_{n}(x)$ 
is unconditionally convergent for all $x \in X$. The proof also shows that if $X$ is infinite dimensional and $c_{0} \hookrightarrow L(X, Y)$, then $\ell_{\infty} \hookrightarrow L(X, Y)[4, \mathbf{1 3}, \mathbf{1 8}]$.

The focus in the opening portion of this paper has dealt with applications of measure theory to problems involving spaces of operators. In the remainder of this paper, the roles are reversed.

If $E$ is separable and $F$ is the dual of a separable space, then there are countably many continuous linear functionals that separate the points in $L_{w^{*}}\left(E^{*}, F\right)$. To see this, suppose that $Y$ is separable, $F=Y^{*}$ and let $L, T \in L_{w^{*}}\left(E^{*}, F\right), L \neq T$. Let $\left(y_{k}\right)$ be dense in $Y$ and consider $\left(y_{k}\right) \subseteq Y^{* *}=F^{*}$. Choose $k \in \mathbb{N}$ so that $L^{*}\left(y_{k}\right) \neq T^{*}\left(y_{k}\right)$. Let $\left\{x_{n}: n \geq 1\right\}$ be dense in $E,\left\|x_{n}\right\|=1, n \in \mathbb{N}$. For each $n$, choose $x_{n}^{*} \in E^{*}$ so that $\left\|x_{n}^{*}\right\|=1$ and $x_{n}^{*}\left(x_{n}\right)=\left\|x_{n}\right\|$. Now let $\left(x_{n_{i}}\right)$ be a subsequence of $\left(x_{n}\right)$ so that $\| x_{n_{i}}-$ $\left(L^{*}\left(y_{k}\right)-T^{*}\left(y_{k}\right)\right) \| \stackrel{i}{\rightarrow} 0$. Therefore $\left\langle x_{n_{i}}^{*}, L^{*}\left(y_{k}\right)-T^{*}\left(y_{k}\right)\right\rangle \neq 0$ for large $i$.

THEOREM 16. Suppose that $\Sigma$ is a $\sigma$-algebra of subsets of $\Omega, E$ is separable and $F$ is a Banach space which does not contain $\ell_{\infty}$ but does embed isomorphically in the dual of a separable space. If $\mu: \Sigma \rightarrow L_{w^{*}}\left(E^{*}, F\right)$ is bounded and finitely additive and $\left(A_{n}\right)$ is a pairwise disjoint sequence from $\Sigma$, then there is a subsequence $\left(B_{n}\right)$ of $\left(A_{n}\right)$ so that

$$
\mu: \sigma\left(\left(B_{n}\right)\right) \rightarrow\left(L_{w^{*}}\left(E^{*}, F\right), \text { sot }\right)
$$

is countably additive.

Proof. Suppose that $\mathcal{P}$ is the power class of the positive integers and identify $\mathcal{P}$ with $\sigma\left(\left(A_{i}\right)\right)$. For $x^{*} \in E^{*}$ and $y^{*} \in F^{*}$, define $F_{x^{*}}: \mathcal{P} \rightarrow F$ by $F_{x^{*}}(A)=\mu(A) x^{*}$ and $G_{y^{*}}: \mathcal{P} \rightarrow E$ by $G_{y^{*}}(A)=\mu(A)^{*} y^{*}$. Since $\ell_{\infty} \hookrightarrow F, F_{x^{*}}$ is strongly additive for each $x^{*} \in E^{*}$ (by the Diestel-Faires theorem). Hence, $\sum \mu(\{n\}) x^{*}$ converges unconditionally for each $x^{*} \in E^{*}$. Since $E$ is separable, $\ell_{\infty} \hookrightarrow E$; thus $G_{y^{*}}$ is strongly additive and $\sum \mu(\{n\})^{*} y^{*}$ converges unconditionally for each $y^{*} \in F^{*}$.

Let $Y$ be separable and $L: F \rightarrow Y^{*}$ be an isomorphism. Define $v: \mathcal{P} \rightarrow$ $L_{w^{*}}\left(E^{*}, Y^{*}\right)$ by

$$
v(A) x^{*}=L\left(\mu(A) x^{*}\right)-L\left(\sum_{n \in A} \mu(\{n\}) x^{*}\right),
$$

for $A \in \mathcal{P}$ and $x^{*} \in E^{*}$. Note that $\nu(A)$ is $w^{*}-w$ continuous since $\sum \mu(\{n\})^{*} y^{*}$ converges unconditionally for each $y^{*} \in F^{*}$. Further, $v$ is a well defined, bounded, finitely additive measure, and $v(\{n\})=0$ for each $n \in \mathbb{N}$. Hence, Lemma 4 applies, and we obtain an infinite subset $M$ of $\mathbb{N}$ such that $v(A)=0$ for all $A \subseteq M$. Then

$$
\mu(A) x^{*}=\sum_{n \in A} \mu(\{n\}) x^{*},
$$

for each $A \subseteq M$ and $x^{*} \in E^{*}$. Thus,

$$
\mu: \sigma\left(\left(A_{n}\right)_{n \in M}\right) \rightarrow\left(L_{w^{*}}\left(E^{*}, F\right)(s o t)\right.
$$

is countably additive.

COROLlaRY 17. Suppose that $\Sigma$ is a $\sigma$-algebra of subsets of $\Omega, E^{*}$ is separable, and $F$ is a Banach space which does not contain $\ell_{\infty}$ but does embed isomorphically in the dual 
of a separable space. If $\mu: \Sigma \rightarrow W(E, F)$ is bounded and finitely additive and $\left(A_{i}\right)$ is a pairwise disjoint sequence from $\Sigma$, then there is a subsequence $\left(B_{i}\right)$ of $\left(A_{i}\right)$ so that

$$
\mu: \sigma\left(\left(B_{i}\right)\right) \rightarrow(W(E, F), \text { sot })
$$

is countably additive.

Proof. Apply Theorem 16 and the isometries stated before Theorem 5.

The next result is stated in two parts for clarity and emphasis. The proof of this theorem is short and straightforward. Further, it facilitates new proofs of the VitaliHahn-Saks-Nikodym theorem, the Nikodym Boundedness theorem and a Banach space version of the Phillips Lemma.

THEOREM 18. Suppose that $\Sigma$ is a $\sigma$-algebra of subsets of $\Omega$.

(i) If $\ell_{\infty}$ does not embed in $E, \ell_{\infty}$ does not embed in $F, \mu: \Sigma \rightarrow K_{w^{*}}\left(E^{*}, F\right)$ is bounded and finitely additive, and $\left(A_{n}\right)$ is a pairwise disjoint sequence from $\Sigma$, then there is a subsequence $\left(B_{n}\right)$ of $\left(A_{n}\right)$ so that

$$
\mu: \sigma\left(\left(B_{n}\right)\right) \rightarrow K_{w^{*}}\left(E^{*}, F\right)
$$

is countably additive.

(ii) If $\ell_{1}$ is not complemented in $E, \ell_{\infty}$ does not embed in $F, \mu: \Sigma \rightarrow K(E, F)$ is bounded and finitely additive, and $\left(A_{n}\right)$ is a pairwise disjoint sequence from $\Sigma$, then there is a subsequence $\left(B_{n}\right)$ of $\left(A_{n}\right)$ so that

$$
\mu: \sigma\left(\left(B_{n}\right)\right) \rightarrow K(E, F)
$$

is countably additive.

Proof. (i) Theorem 5 implies that $\ell_{\infty} \hookrightarrow K_{w^{*}}\left(E^{*}, F\right)$. Then $\mu$ is strongly additive (by the Diestel-Faires theorem), and the conclusion follows from Drewnowski's result stated in the Introduction.

(ii) Apply part (i) and the isometries stated before Theorem 5.

Corollary 19. (i) Suppose that $\Sigma$ is a $\sigma$-algebra, $\ell_{\infty}$ does not embed in $X$, and $Y$ is any Banach space. If $\mu: \Sigma \rightarrow K_{w^{*}}\left(X^{*}, Y\right)$ is a measure which is countably additive in the weak operator topology, then $\mu$ is norm countably additive.

(ii) (16, Theorem 5) Suppose that $\Sigma$ is a $\sigma$-algebra, $\ell_{1}$ is not complemented in $X$, and $Y$ is any Banach space. If $\mu: \Sigma \rightarrow K(X, Y)$ is a measure which is countably additive in the weak operator topology, then $\mu$ is norm countably additive.

Proof. (i) Suppose that $\mu: \Sigma \rightarrow K_{w^{*}}\left(X^{*}, Y\right)$ is countably additive in the (wot) and $\mu$ is not countably additive. Then there is a $p>0$ and a pairwise disjoint sequence $\left(A_{i}\right)$ so that $\left\|\mu\left(A_{i}\right)\right\|>p$ for all $i$. Let $Z=\left[\mu\left(A_{i}\right)\left(X^{*}\right): i \geq 1\right]$. Since $\mu$ is countably additive in the $(w o t), \mu(B)\left(X^{*}\right) \subseteq Z$ for all $B \in \sigma\left(\left(A_{i}\right)\right)$. Note that $\mu\left(A_{i}\right)\left(X^{*}\right)$ is separable for each $i$ (since $\mu\left(A_{i}\right)$ is compact), and thus $Z$ is separable. Apply Theorem 18 to find a subsequence $\left(B_{i}\right)$ of $\left(A_{i}\right)$ so that $\mu: \sigma\left(\left(B_{i}\right)\right) \rightarrow K_{w^{*}}\left(X^{*}, Z\right)$ is countably additive. But $\left\|\mu\left(B_{i}\right)\right\|>p$ for all $i$, a contradiction.

(ii) Apply part (i) and the isometries stated before Theorem 5.

COROLlary 20. Suppose that $\Sigma$ is a $\sigma$-algebra of subsets of $\Omega$. 
(i) If $\ell_{\infty} \hookrightarrow X, \mu: \Sigma \rightarrow X$ is a bounded finitely additive set function, and $\left(A_{n}\right)$ is a pairwise disjoint sequence in $\Sigma$, then there is a sub -sequence $\left(B_{n}\right)$ of $\left(A_{n}\right)$ such that $\mu$ is countably additive on $\sigma\left(\left(B_{n}\right)\right)$.

(ii) ([5, p. 79]). If $\mu \in b a(\Sigma)$ and $\left(A_{n}\right)$ is a pairwise disjoint sequence in $\Sigma$, then there is a subsequence $\left(B_{n}\right)$ of $\left(A_{n}\right)$ such that $\mu$ is countably additive on $\sigma\left(\left(B_{n}\right)\right)$.

Proof. (i) We note that $K(\mathbb{R}, X)=L(\mathbb{R}, X) \simeq X$. Apply Theorem 18 .

Corollary 21 (Vitali-Hahn-Saks-Nikodym, [6, p. 23]). Suppose that $\Sigma$ is a $\sigma$-algebra of subsets of $\Omega, \mu_{n}: \Sigma \rightarrow X$ is strongly additive for each $n$, and $\mu(A)=$ $\lim _{n} \mu_{n}(A)$ exists for each $A \in \Sigma$. Then

$$
\lim _{i} \sup _{n}\left\|\mu_{n}\left(A_{i}\right)\right\|=0,
$$

whenever $\left(A_{i}\right)$ is a pairwise disjoint sequence from $\Sigma$, i.e. $\left(\mu_{n}\right)$ is uniformly strongly additive. Consequently, $\mu$ is strongly additive.

Proof. Suppose not. Then there exists an $\epsilon>0$ and a sequence of pairwise disjoint sets $\left(A_{n}\right)$ in $\Sigma$ such that $\left\|\mu_{n}\left(A_{n}\right)\right\|>\epsilon$ for each $n$. Let $\Sigma_{1}=\sigma\left(\left(A_{n}\right)\right), U=\left\{\mu_{n}\left(A_{i}\right): n \geq\right.$ $1, i \geq 1\}$, and $Y=[U]$. Then $Y$ is a separable subspace of $X$ (since $\mathrm{U}$ is countable). Further, if $A \in \Sigma_{1}$, then $\mu_{n}(A) \in Y$ for each $n$. By Drewnowski's result quoted in the Introduction and a standard diagonalisation argument, we may assume that each $\mu_{n}: \Sigma_{1} \rightarrow Y$ is countably additive. For each $n \in \mathbb{N}$, let $x_{n}^{*} \in Y^{*}$ of norm one so that $\left|x_{n}^{*} \mu_{n}\left(A_{n}\right)\right|=\left\|\mu_{n}\left(A_{n}\right)\right\|>\epsilon$. Denote the Hahn-Banach extension of $x_{n}^{*}$ in $X^{*}$ also by $x_{n}^{*}$. Since $Y$ is separable, using a Cantor diagonalisation argument, we obtain a subsequence $\left(y_{n}^{*}\right)$ of $\left(x_{n}^{*}\right)$ so that $\lim _{n} y_{n}^{*}(y)$ exists for each $y \in Y$.

Therefore $\left(y_{n}^{*} \mu_{n}\right)$ is a sequence of scalar valued countably additive measures and $\lim _{n} y_{n}^{*} \mu_{n}(A)$ exists for each $A \in \Sigma_{1}$. Let $c$ denote the space of all convergent sequences of real numbers equipped with the sup norm. Now define $\tau: \Sigma_{1} \rightarrow c$ by

$$
\tau(A)=\left(y_{n}^{*} \mu_{n}(A)\right)_{n}
$$

for $A \in \Sigma_{1}$. Note that $c$ is separable and $c \simeq L(\mathbb{R}, c)$. By Theorem 18, there is a subsequence $\left(B_{i}\right)$ of $\left(A_{i}\right)$ so that $\tau: \sigma\left(\left(B_{i}\right)\right) \rightarrow L(\mathbb{R}, c)=K(\mathbb{R}, c)$ is countably additive. But $\left\|\tau\left(B_{n}\right)\right\| \geq\left|y_{n}^{*} \mu_{n}\left(B_{n}\right)\right|>\epsilon$, a contradiction.

The strong additivity of $\mu$ follows from the uniform strong additivity of $\left(\mu_{n}\right)$ and the fact that $\left(\mu_{n}(A)\right) \rightarrow \mu(A)$ for each $A$.

Corollary 22 (The Nikodym Boundedness theorem, [6, p. 14]). Suppose that $\Sigma$ is a $\sigma$-algebra of subsets of $\Omega$ and $\left(\mu_{n}\right)$ is a sequence of $X$-valued bounded vector measures. If $\sup \left\{\left\|\mu_{n}(A)\right\|: n \in \mathbb{N}\right\}<\infty$ for each $A \in \Sigma$, then $\left(\mu_{n}\right)$ is uniformly bounded, i.e.

$$
\sup \left\{\left\|\mu_{n}(A)\right\|: n \in \mathbb{N}, A \in \Sigma\right\}<\infty .
$$

Proof. By replacing $\left\{\mu_{n}: n \in \mathbb{N}\right\}$ with $\left\{x^{*} \mu_{n}: n \in \mathbb{N}, x^{*} \in X^{*},\left\|x^{*}\right\| \leq 1\right\}$, it is enough to prove the theorem for sequences of real-valued measures. Now suppose that $\left(\mu_{n}\right)$ is a sequence of real-valued finitely additive measures such that $\sup _{n}\left|\mu_{n}(A)\right|<\infty$ for each $A$. Note that, since $\mu_{n}$ is a bounded real-valued measure, $\mu_{n}$ is strongly additive for each $n$.

By contradiction, suppose that $\sup \left\{\left|\mu_{n}(A)\right|: n \in \mathbb{N}, A \in \Sigma\right\}=\infty$. We claim that for each $M>0$, there is an $n \in \mathbb{N}$ and a partition $\{A, B\}$ of $\Omega$ into pairwise disjoint 
members of $\Sigma$ such that $\left|\mu_{n}(A)\right|>M$ and $\left|\mu_{n}(B)\right|>M$. Let $n \in \mathbb{N}$ and $A \in \Sigma$ so that $\left|\mu_{n}(A)\right|>M+\sup _{i}\left|\mu_{i}(\Omega)\right|$. Then

$$
\left|\mu_{n}(\Omega \backslash A)\right|=\left|\mu_{n}(A)-\mu_{n}(\Omega)\right| \geq\left|\mu_{n}(A)\right|-\left|\mu_{n}(\Omega)\right|>M .
$$

Then the sets $A$ and $B=\Omega \backslash A$ satisfy the claim.

Let $M=1$ and choose $n_{1} \in \mathbb{N}$ and $\left\{A_{1}, B_{1}\right\}$ a partition of $\Omega$ such that $\left|\mu_{n_{1}}\left(A_{1}\right)\right|>1$ and $\left|\mu_{n_{1}}\left(B_{1}\right)\right|>1$. Now, either $\sup \left\{\left|\mu_{n}\left(A \cap A_{1}\right)\right|: n \in \mathbb{N}, A \in \Sigma\right\}=\infty$ or $\sup \left\{\mid \mu_{n}(A \cap\right.$ $\left.\left.B_{1}\right) \mid: n \in \mathbb{N}, A \in \Sigma\right\}=\infty$. Otherwise, $\sup \left\{\left|\mu_{n}(A)\right|: n \in \mathbb{N}, A \in \Sigma\right\}<\infty$. Without loss of generality, suppose that

$$
\sup \left\{\left|\mu_{n}\left(A \cap A_{1}\right)\right|: n \in \mathbb{N}, A \in \Sigma\right\}=\infty .
$$

Let $C_{1}=A_{1}$ and $D_{1}=\Omega \backslash C_{1}$.

Now treat $C_{1}$ as $\Omega$ and choose an $n_{2}>n_{1}$ and a partition $\left\{A_{2}, B_{2}\right\}$ of $C_{1}$ such that $\left|\mu_{n_{2}}\left(A_{2}\right)\right|>2,\left|\mu_{n_{2}}\left(B_{2}\right)\right|>2$, and without loss of generality

$$
\sup \left\{\left|\mu_{n}\left(A \cap A_{2}\right)\right|: n \in \mathbb{N}, A \in \Sigma\right\}=\infty .
$$

Let $C_{2}=A_{2}$ and $D_{2}=C_{1} \backslash A_{2}$.

Continue this process and obtain an increasing subsequence $\left(n_{i}\right)$ of positive integers and a pairwise disjoint sequence of sets $\left(D_{i}\right)$ such that $\left|\mu_{n_{i}}\left(D_{i}\right)\right|>i$ for each $i$. Relabelling, we may assume that $\left|\mu_{i}\left(D_{i}\right)\right|>i$ for each $i$.

Define $v_{i}(A)=\frac{1}{i} \mu_{i}(A)$, for $A \in \Sigma, i \in \mathbb{N}$. Then $v_{i}: \Sigma \rightarrow \mathbb{R}$ is strongly additive for each $i$ and $\lim _{i} v_{i}(A)=0$ for each $A$. By the Vitali-Hahn-Saks-Nikodym theorem, $\left(v_{i}\right)$ is uniformly strongly additive, and thus $\lim _{i}\left|v_{i}\left(D_{i}\right)\right|=0$. However, $\left|v_{i}\left(D_{i}\right)\right|>1$ for each $i$, a contadiction.

If $F$ is a separable Banach space, then the space $c(F)=\left\{\left(y_{n}\right): n \in \mathbb{N}\right\} \subseteq F^{\mathbb{N}}$ of all convergent sequences in $F$ is separable when endowed with the usual pointwise operations and the sup norm. Let $c_{0}(F)$ be the subspace of $c(F)$ consisting of null sequences. Note that $c_{0}(F) \simeq L\left(\mathbb{R}, c_{0}(F)\right)=K\left(\mathbb{R}, c_{0}(F)\right)$.

Corollary 23 (Banach space version of the Phillips Lemma). If $\mu_{n}: \mathcal{P} \rightarrow X$ is strongly additive (in norm) for each $n$, and $\left(\mu_{n}(A)\right) \rightarrow 0$ for each $A \in \mathcal{P}$, then

$$
\lim _{n} \sum_{k \in A} \mu_{n}(\{k\})=0
$$

uniformly for $A \in \mathcal{P}$.

Proof. Suppose not. Choose $\epsilon>0,\left(A_{i}\right)$ a sequence in $\mathcal{P}$, and $\left(\lambda_{i}\right)$ a subsequence of $\left(\mu_{i}\right)$ such that $\left\|\sum_{k \in A_{i}} \lambda_{i}(\{k\})\right\|>2 \epsilon$ for each $i \in \mathbb{N}$. Using the unconditional convergence of $\sum_{k} \lambda_{i}(\{k\})$ (by the strong additivity of $\left.\lambda_{i}\right)$ and the fact that $\left\|\mu_{n}(A)\right\| \rightarrow 0$ for each $A$, we can find an increasing sequence $\left(N_{i}\right)$ of natural numbers and a subsequence $\left(\lambda_{n_{i}}\right)$ of $\left(\lambda_{i}\right)$ such that

$$
\left\|\sum_{k \in A_{n_{i}} \cap\left[N_{i-1}, N_{i}\right)} \lambda_{n_{i}}(\{k\})\right\|>\epsilon
$$

for each $i \in \mathbb{N}$. Let $B_{i}=A_{n_{i}} \cap\left[N_{i-1}, N_{i}\right), \Sigma=\sigma\left(\left(B_{i}\right)\right)$ and $v_{i}=\lambda_{n_{i}}$. If $U=\left\{v_{i}\left(B_{j}\right): i \geq\right.$ $1, j \geq 1\}$, then $Y=[U]$ is separable (since $B_{i}$ are pairwise disjoint), hence $c(Y)$ and 
$c_{0}(Y)$ are separable. Define

$$
v(A)=\left(v_{i}(A)\right)_{i}
$$

for $A \in \Sigma$. If $A \in \Sigma$, then $v(A) \in c_{0}(Y)$. Applying Theorem 18 to $v: \Sigma \rightarrow L\left(\mathbb{R}, c_{0}(Y)\right)$, one obtains a subsequence $\left(C_{i}\right)$ of $\left(B_{i}\right)$ so that $v$ is countably additive on $\sigma\left(\left(C_{i}\right)\right)$. However, $\left\|\sum_{k \in C_{i}} v_{i}(\{k\})\right\|>\epsilon$ for each $i$, a contradiction.

Corollary 24 (A generalisation of a result of Schur, [1]). Suppose that $X$ is a Banach space which does not contain an isomorphic copy of $\ell_{\infty}$. Suppose further that $\left(x_{i n}\right)$ is a doubly indexed sequence in $X$ so that

(a) $\sum_{n=1}^{\infty} x_{i n}$ is unconditionally convergent for each $i$, and

(b) $\lim _{i} \sum_{n \in A} x_{i n}$ exists for all $A \subseteq \mathbb{N}$.

If $x_{n}=\lim _{i} x_{\text {in }}$ for each $n$, then

$$
\lim _{i} \sum_{n \in A}\left(x_{i n}-x_{n}\right)=0
$$

uniformly for $A \subseteq \mathbb{N}$.

Proof. Let $v(A)=\lim _{i} \sum_{n \in A} x_{i n}$, for $A \subseteq \mathbb{N}$. Then $v$ is finitely additive and the Nikodym Boundedness theorem ensures that $v$ is bounded. Furthermore, since $\ell_{\infty} \hookrightarrow$ $X, v$ must be strongly additive (by the Diestel-Faires theorem). Define $\mu_{i}: \mathcal{P} \rightarrow X$ by

$$
\mu_{i}(A)=\sum_{n \in A} x_{i n}-v(A) .
$$

Therefore $\left(\mu_{i}(A)\right) \rightarrow 0$ for each $A \subseteq \mathbb{N}$ and $\mu_{i}$ is strongly additive for each $i$. Apply Phillips's Lemma to conclude that

$$
\lim _{i} \sum_{n \in A} \mu_{i}(\{n\})=\lim _{i} \sum_{n \in A}\left(x_{i n}-x_{n}\right)=0
$$

uniformly for $A \subseteq \mathbb{N}$.

If $\Sigma$ is a $\sigma$-algebra of subsets of $\Omega$, then $c a(\Sigma)$ denotes the Banach space (with total variation norm) of countably additive real measures $\mu: \Sigma \rightarrow \mathbb{R}$. If $X$ is infinite dimensional, then there exists a bounded and countably additive measure $\mu$ defined on a $\sigma$-algebra with values in $X$ with infinite total variation. Suppose that $X$ is infinite dimensional and let $\sum x_{n}$ be an unconditionally convergent series in $X$ which is not absolutely summable. Define $\mu: \mathcal{P} \rightarrow X$ by $\mu(A)=\sum_{n \in A} x_{n}$, if $A \subseteq \mathbb{N}$. Then $\mu$ is countably additive and does not have finite variation since the infinite series is not absolutely summable. $c a(\Sigma, X)$ denotes the Banach space (with sup norm) of countably additive vector measures $\mu: \Sigma \rightarrow X$, and $\operatorname{cabv}(\Sigma, X)$ denotes the Banach space (with total variation norm) of countably additive measures $\mu: \Sigma \rightarrow X$ with bounded total variation. For $\mu \in \operatorname{cabv}(\Sigma, X),|\mu|$ denotes the total variation of $\mu$. A subset $S$ of $X$ is said to be weakly precompact provided that every bounded sequence from $S$ has a weakly Cauchy subsequence.

COROLlARY 25. (i) If $K$ is a weakly precompact subset of $c a(\Sigma)$, then $K$ is uniformly strongly additive, i.e. if $\left(A_{i}\right)$ is a pairwise disjoint sequence in $\mathcal{A}$, then $\lim _{i}\left|\mu\left(A_{i}\right)\right|=0$ uniformly for $\mu \in K$. 
(ii) If $K$ is a weakly precompact subset of $c a(\Sigma, X)$ and $X$ is a Schur space, then $K$ is uniformly strongly additive, i.e. if $\left(A_{i}\right)$ is a pairwise disjoint sequence in $\mathcal{A}$, then $\lim _{i}\left\|\mu\left(A_{i}\right)\right\|=0$ uniformly for $\mu \in K$.

(iii) If $K$ is a weakly precompact subset of $\operatorname{cabv}(\Sigma, X)$, then $|K|=\{|\mu|: \mu \in K\}$ is uniformly strongly additive.

Proof. (i) Suppose that $\left(\mu_{n}\right)$ is a sequence from $K$, and without loss of generality suppose that $\left(\mu_{n}\right)$ is weakly Cauchy. Now, weakly Cauchy sequences in $c a(\Sigma)$ are weakly convergent [5, p. 91]. Suppose $\left(\mu_{n}\right)$ converges weakly to $\mu \in c a(\Sigma)$. Then $\mu_{n}(A) \rightarrow$ $\mu(A)$ for each $A \in \Sigma$. Note that each $\mu_{n}$ is strongly additive. The Vitali-Hahn-SaksNikodym theorem implies that $\left(\mu_{n}\right)$ is uniformly strongly additive. If $\left(A_{i}\right)$ is a pairwise disjoint sequence from $\Sigma$, then $\lim _{i} \sup _{n}\left|\mu_{n}\left(A_{i}\right)\right|=0$.

(i) Suppose that $\left(\mu_{n}\right)$ is a sequence from $K$, and without loss of generality suppose that $\left(\mu_{n}\right)$ is weakly Cauchy. Then for each $A \in \Sigma,\left(\mu_{n}(A)\right)$ is weakly Cauchy in $X$. Since $X$ is a Schur space, $\mu(A)=\lim _{n} \mu_{n}(A)$ exists for each $A \in \Sigma$. The Vitali-HahnSaks-Nikodym theorem implies that $\left(\mu_{n}\right)$ is uniformly strongly additive, i.e. if $\left(A_{i}\right)$ is a pairwise disjoint sequence from $\Sigma$, then $\lim _{i} \sup _{n}\left\|\mu_{n}\left(A_{i}\right)\right\|=0$.

(iii) Suppose that $K$ is a weakly precompact subset of $\operatorname{cabv}(\Sigma, X)$ and $|K|=\{|\mu|$ : $\mu \in K\}$ is not uniformly strongly additive. Then one obtains $p>0$, a sequence $\left(\mu_{i}\right)$ in $K$, and a pairwise disjoint sequence $\left(A_{i}\right)$ in $\Sigma$ such that $\left|\mu_{i}\right|\left(A_{i}\right)>p$ for each $i$. For each $i$, let $\left(A_{i j}\right)_{j=1}^{n_{i}}$ be a finite partition of $A_{i}$ and $\left(x_{i j}^{*}\right)_{j=1}^{n_{i}}$ in $B_{X^{*}}$ such that

$$
\sum_{j=1}^{n_{i}}\left|x_{i j}^{*} \mu_{i}\left(A_{i j}\right)\right|>p .
$$

Define $T: \operatorname{cabv}(\Sigma, X) \rightarrow \ell_{1}$ by

$$
T(v)=\left(x_{11}^{*} v\left(A_{11}\right), \ldots, x_{1 n_{1}}^{*} v\left(A_{1 n_{1}}\right), x_{21}^{*} v\left(A_{21}\right), \ldots, x_{2 n_{2}}^{*} v\left(A_{2 n_{2}}\right), x_{31}^{*} v\left(A_{31}\right), \ldots\right) .
$$

Note that $\|T(v)\| \leq|v|$ for all $v \in \operatorname{cabv}(\Sigma, X)$ and $T$ is a continuous linear map. Since $T(K)$ is a weakly precompact subset of $\ell_{1} \simeq \operatorname{cabv}(\mathcal{P}, \mathbb{R})$, by (i) it follows that $T(K)$ is uniformly strongly additive. However, this is a contradiction with (1).

\section{REFERENCES}

1. S. Banach, Theorie des operations linéaires (Monografie Matematyczme, Warsaw, 1932).

2. E. Bator and P. Lewis, Complemented spaces of operators, Bull. Pol. Acad. Sci. Math. 50(4) (2002), 413-416.

3. E. M. Bator, P. W. Lewis and D. R. Slavens, Vector measures, $c_{0}$, and (sb) operators, Bull. Pol. Acad. Sci. Math. 54(1) (2006), 63-73.

4. C. Bessaga and A. Pelczynski, On bases and unconditional convergence of series in Banach spaces, Studia Math. 17 (1958), 151-174.

5. J. Diestel, Sequences and series in Banach spaces, Graduate Texts in Mathematics, no. 92 (Springer-Verlag, Berlin, 1984).

6. J. Diestel and J. J. Uhl, Jr., Vector measures, Mathematical Surveys 15 (American Mathematical Society, Providence, RI, 1977).

7. L. Drewnowski, Copies of $\ell_{\infty}$ in an operator space, Math. Proc. Camb. Phil. Soc. 108 (1990), 523-526.

8. G. Emmanuele, Remarks on the uncomplemented subspace $W(E, F), J$. Funct. Anal. 99 (1991), 125-130.

9. G. Emmanuele, A remark on the containment of $c_{0}$ in spaces of compact operators, Math. Proc. Camb. Phil. Soc. 111 (1992), 331-335. 
10. G. Emmanuele, About the position of $K_{w^{*}}\left(X^{*}, Y\right)$ inside $L_{w^{*}}\left(X^{*}, Y\right)$, Atti. Sem. Mat. Fis. Univ. Modena 42(1) (1994), 123-133.

11. M. Feder, On the non-existence of a projection onto the space of compact operators, Canad. Math. Bull. 25 (1982), 78-81.

12. I. Ghenciu, Complemented spaces of operators, Proc. Amer. Math. Soc. 133(9) (2005), 2621-2623.

13. I. Ghenciu and P. Lewis, The embeddability of $c_{0}$ in spaces of operators, Bull. Pol. Acad. Sci. Math. 56(3-4) (2008), 239-256.

14. I. Ghenciu and P. Lewis, Dunford-Pettis properties and spaces of operators, Canad. Math. Bull. 52 (2009), 213-223. 173.

15. K. John, On the uncomplemented subspace K(X, Y), Czech. Math. J. 42 (1992), $167-$

16. N. Kalton, Spaces of compact operators, Math. Ann. 208 (1974), 267-278.

17. J. Kupka, A short proof and generalization of a measure theoretic disjointization lemma, Proc. Amer. Math. Soc. 45(1) (1974), 70-72.

18. P. Lewis, Spaces of operators and $c_{0}$, Studia Math. 145 (2001), 213-218.

19. P. Lewis and P. Schulle, Non-complemented spaces of linear operators, vector measures, and $c_{0}$, Canad. Math. Bull., to appear.

20. A. Pietsch, Nuclear locally convex spaces, Ergebnisse der Mathematik und ihrer Grenzgebiete, Band 66 (Springer-Verlag, New York, 1972).

21. H. R. Pitt, A note on bilinear forms, J. Lond. Math. Soc. 11 (1936), 174-180.

22. H. Rosenthal, On relatively disjoint families of measures, with some applications to Banach space theory, Studia Math. 37 (1970), 13-36.

23. W. Ruess, Duality and geometry of spaces of compact operators, functional analysis: Surveys and recent results III. in Proceedings of 3rd Paderborn Conference 1983, North-Holland Mathematics Studies no. 90 (North-Holland Publ. Co., New York, NY, 1984), 59-78.

24. I. Singer, Bases in Banach spaces (Springer, New Mexico, 1981). 